\title{
Optimal security-constrained power scheduling by Benders decomposition
}

\author{
Jorge Martínez-Crespo ${ }^{\mathrm{a}, *}$, Julio Usaola ${ }^{\mathrm{a}}$, José L. Fernández ${ }^{\mathrm{b}}$ \\ ${ }^{a}$ Department of Electrical Engineering, Universidad Carlos III de Madrid, Avda. Universidad 30, 28911-Leganés, Madrid, Spain \\ ${ }^{\mathrm{b}}$ Regulation Department of Red Eléctrica de España (REE), Madrid, Spain
}

\begin{abstract}
This paper presents a Benders decomposition approach to determine the optimal day-ahead power scheduling in a pool-organized power system, taking into account dispatch, network and security constraints. The study model considers the daily market and the technical constraints resolution as two different and consecutive processes. The daily market is solved in a first stage subject to economical criteria exclusively and then, the constraints solution algorithm is applied to this initial dispatch through the redispatching method. The Benders partitioning algorithm is applied to this constraints solution process to obtain an optimal secure power scheduling.

The constraints solution includes a full AC network and security model to incorporate voltages magnitudes as they are a critical factor in some real power systems. The algorithm determines the active power committed to each generator so as to minimize the energy redispatch cost subject to dispatch, network and security constraints. The solution also provides the reactive power output of the generators, the value of the transformers taps and the committed voltage control devices.

The model has been tested in the IEEE 24-bus Reliability Test System and in an adapted IEEE 118-bus Test System. It is programmed in GAMS mathematical modeling language. Some relevant results are reported.
\end{abstract}

Keywords: Electricity markets; Constraints solution; Preventive security analysis; Benders decomposition

\section{Introduction}

This paper presents an application of Benders decomposition [1,2] to define the optimal security-constrained daily unit commitment $[3,4]$ in a competitive environment. Dispatch constraints (power reserve, ramp rate limits, minimum up and down time) as well as network (full power flow equations) and security constraints (bus voltages and transmission flow limits in pre and post-contingency states) have been included in the study model. This model is based on the structure of the Spanish day-ahead pool-based electricity market [5]. Two separate entities, the Market Operator (MO) and the System Operator (SO) [5], manage the power system operation. The MO is in charge of the operation of the daily electricity market trading, whereas the SO is the entity responsible for the secure operation of the system.

The problem, optimal power scheduling with preventive security criterion, is solved in two different and consecutive stages. In a first stage, the MO carries out a dispatch (daily market) according to economic criteria and without network and security constraints. The objective of the daily market is the maximization of the system net social benefit. In this day-ahead energy market, the market participants submit hourly energy multi-block price bids and the MO sets the accepted bids, the market clearing energy and the hourly market clearing price paid to every committed generating unit.

The grid and security constraints are included in a second stage (technical constraints solution process) performed by the SO, where the security of the daily market solution is checked. The same bids submitted by the generating units to the daily market are used to solve the security problem. For this process, demand-side bids are not considered. If this generation scheduling does not

\footnotetext{
* Corresponding author. Tel.: +3491 6249948; fax: +34 916249430.

E-mail addresses: jorgemar@ing.uc3m.es (J. Martínez-Crespo), jusaola@ing.uc3m.es (J. Usaola), joselfdez@ree.es (J.L. Fernández).
} 
fulfill the power system security criteria [6], the constraints solution process begins. Power system constraints are solved through redispatching method [7], that is, by increasing and decreasing generation of connected units or by committing previously off-line generators, as well as connecting voltage control devices. Some generating units can be committed or de-committed as a result of it. De-committed energy is not paid, whereas new committed energy is paid to the price that it has been offered.

Based on this scheme, this paper proposes an alternative method to define a preventive secure power scheduling. The optimization procedure includes a mixed-integer unit commitment problem [8] and a non-linear security-constrained optimal power flow (SCOPF) $[9,10]$. A preventive security criterion is used, incorporating the commitment of voltage control elements, such as reactors or capacitors.

Therefore, in this work, the daily power scheduling is a complex mathematical problem that includes:

- linear objective function based on hourly energy multi-block price bids;

- binary decision variables (off-line or on-line generating units, reactors or capacitors during period $t$ );

- continuous variables for the operation processes (real and reactive power, transformer taps, ...);

- time couplings (ramp rate limits, minimum up and down time);

- non-linear constraints (complete AC power flow equations, transmission flow limits).

Due to the non-convexities introduced by the binary variables and the non-linear conditions, it is necessary to solve the problem using partitioning methods as Lagrangian Relaxation, a method widely used in the scientific literature [11-14], or the one selected here, generalized Benders decomposition (GBD) $[15,16]$. The time couplings are arranged so that they can be treated in an optimal way by the Benders algorithm.

The method defined in this paper involves a progress with regard to available centralized approaches [15-18] as it provides an optimal and secure time-coupled scheduling considering a precise model of the transmission network and a complete security analysis, solving jointly overloads and voltage constraints and providing an optimal and preventive real power dispatch with voltage control devices. A nested decomposition algorithm based on this method has been applied to a real power system [19]. Nevertheless, due to the huge amount of the variables and constraints involved, the application of these methods to large-scale power systems needs to be improved in the future in order to reduce computation time.

The model has been tested in the IEEE 24-bus Reliability Test System [20] and an adapted IEEE 118-bus System [21]. The execution times and iterations number are compared and some results for the IEEE 24-bus System are reported. The model has been programmed in GAMS [22], using CONOPT [23] and CPLEX [24] solvers for non-linear and linear mixed-integer programming problems, respectively.

This paper is organized in the following way. The notation used throughout the article is provided in nomenclature. The third section expounds the model formulation. The structure of the Benders decomposition algorithm and its application to the optimal secure power scheduling are explained in the fourth section. The following section presents the test systems and some relevant results, comparing time-coupled and decoupled solutions and finally, the last section states the conclusions.

\section{Problem formulation}

After the liberalization of the electricity industry, in most of countries, the unit commitment problem is solved as a market problem based on bid prices, instead of the cost-based minimization of the classical model. The approach in this paper is based on the Spanish electricity market rules [5]. These rules establish that all available generating units must submit power bids arranged in different blocks with their own price. The same power bids are used in the daily market and the constraints solution process.

The MO performs an initial daily market without network constraints, which settles the market clearing price. Starting from this power dispatch and before the constraints solution stage, a contingency analysis is carried out by the SO to define those potential outage events that would cause overloads or out-of-limits voltages. Once these contingencies have been selected, they are included in the study model and corrections are made to this first dispatch in the technical constraints solution process to fulfill network and security limits.

\subsection{Daily market}

In this phase, the dispatcher (MO) problem is to select the cheapest offers from the set of the supply-side offers to match the real power demand (demand-side bids are not considered in this study ${ }^{1}$ ). All the committed generating units are paid at the market clearing price. Therefore, the objective is to minimize the 24 hourly marginal prices, which is equivalent to minimize the total generation cost.

\footnotetext{
${ }^{1}$ The majority of the energy purchase offers follow a rigid demand pattern and they are not used in the constraints solution procedure, main objective of this paper. For these reasons, the demand-side offers have not been incorporated in the formulation.
} 
Mathematically, the objective function can be stated as the minimization of:

$$
\sum_{t \in T} \sum_{i \in G} \sum_{b \in B} p_{t, i, b}^{\bullet} \cdot P_{t, i, b}
$$

The objective function does not include fixed costs or start-up costs, only energy bids divided in blocks $\left(P_{t, i, b}\right)$ as well as their bid prices, $p_{t, i, b}^{\bullet}$. For simplicity, it is assumed that the minimum power of thermal plant, $P_{i}^{\text {min }}$, is always offered as the first block, which is considered as indivisible energy block through the decision binary variable $u_{t, i}$. The acceptance of this first block involves the unit start-up. With this consideration, the objective function (1) could be formulated as:

$$
\sum_{t \in T} \sum_{i \in G} p_{t, i, 1}^{\bullet} \cdot u_{t, i} \cdot P_{i}^{\min }+\sum_{t \in T i \in G} \sum_{\substack{b \in B \\ b>1}} p_{t, i, b}^{\bullet} \cdot P_{t, i, b}
$$

The objective function will be subject to the following constraints:

(a) Energy blocks limits:

$$
0 \leq P_{t, i, b} \leq P_{t, i, b}^{\max } \quad \forall t \in T, \forall i \in G, \forall(b>1) \in B
$$

(b) Real power output limits:

$$
u_{t, i} \cdot P_{i}^{\min } \leq P_{t, i} \leq u_{t, i} \cdot P_{i}^{\max } \quad \forall t \in T, \forall i \in G
$$

(c) Relation between energy blocks and real power output:

$$
P_{t, i}=u_{t, i} \cdot P_{i}^{\min }+\sum_{\substack{b \in B \\ b>1}} P_{t, i, b} \quad \forall t \in T, \forall i \in G
$$

(d) Real power balance:

$$
\sum_{j \in N} P_{t, j}^{d}+P_{t}^{\text {loss }}=\sum_{i \in G} P_{t, i} \quad \forall t \in T
$$

(e) Ramp rate limits:

$$
-\mathrm{DR}_{i} \leq P_{t, i}-P_{t-1, i} \leq \mathrm{UR}_{i} \quad \forall t \in T, \forall i \in G
$$

In this process, the generation is only approximate, since an initial estimation of the system losses, $P_{t}^{\text {loss }}$, is included. The market clearing price per period $\tilde{p}_{t}$ is obtained as outcome of the minimization and it is equal to the price of the last energy block accepted to match the active power demand for each period.

\subsection{Technical constraints solution process}

Once after the wholesale daily market has been held, the security conditions are verified by the SO. If the scheduling resulting from the spot market does not meet the security requirements, the optimization algorithm will modify the preceding units scheduling using the energy multi-block price bids submitted to the daily market. This new generation will be paid according to its bid price.

Therefore, the objective function to minimize is:

$$
\sum_{t \in T} \sum_{i \in \mathrm{CG}} \sum_{\substack{b \in B \\ b>1}} p_{t, i, b}^{\bullet} \cdot \Delta P_{t, i, b}^{\mathrm{up}}+\sum_{t \in T} \sum_{i \in \mathrm{UG}} p_{t, i, 1}^{\bullet} \cdot u_{t, i}^{\mathrm{on}} \cdot P_{t, i}^{\mathrm{min}}+\sum_{t \in T} \sum_{i \in \mathrm{UG}} \sum_{b \in B} p_{t, i, b}^{\bullet} \cdot \Delta P_{t, i, b}^{\mathrm{up}}
$$

The first term of the objective function represents the overcost of real power increase for the committed generating units in the spot market. The second and third terms mean the overcost of new committed units. The second term involves the 'start-up' cost of a new generating unit.

It should be noted that the SO only defines the variation of generation needed to eliminate power system constraints. The generation-demand balance with the new committed energy is established by the power flow equations.

The MO defines the final units scheduling in a latter process. The MO modifies the initial market clearing including the re-dispatch provided by the SO, following the precedence order of the bids submitted by the generators into the market (if some energy have to be retired), and applying certain heuristic rules, e.g., in case of the same energy bid prices for different units. This last adjustment is not added to the constraints solution model because, as it was previously mentioned, this retired energy is not paid and because this process is based on some heuristic criteria. 
Besides Eq. (7), the constraints included in this stage are:

(a) Power redispatch blocks limits:

$$
\begin{aligned}
& 0 \leq \Delta P_{t, i, b}^{\text {up }} \leq P_{t, i, b}^{\max } \quad \forall t \in T, \forall i \in G, \forall(b>1) \in B \\
& 0 \leq \Delta P_{t, i, b}^{\text {down }} \leq P_{t, i, b}^{\max } \quad \forall t \in T, \forall i \in G, \forall(b>1) \in B
\end{aligned}
$$

(b) Real power output by the units previously committed in the daily market:

$$
\begin{aligned}
& \left(1-u_{t, i}^{\text {off }}\right) \cdot P_{i}^{\text {min }} \leq P_{t, i} \leq\left(1-u_{t, i}^{\text {off }}\right) \cdot P_{i}^{\max } \\
& P_{t, i}=P_{t, i}^{0}-u_{t, i}^{\text {off }} \cdot P_{i}^{\text {min }}+\sum_{\substack{b \in B \\
b>1}} \Delta P_{t, i, b}^{\text {up }}-\sum_{\substack{b \in B \\
b>1}} \Delta P_{t, i, b}^{\text {down }} \quad \forall t \in T, \forall i \in \mathrm{CG}
\end{aligned}
$$

(c) Real power output by the units committed in this new stage:

$$
\begin{aligned}
& u_{t, i}^{\mathrm{on}} \cdot P_{i}^{\mathrm{min}} \leq P_{t, i} \leq u_{t, i}^{\mathrm{on}} \cdot P_{i}^{\mathrm{max}} \\
& P_{t, i}=u_{t, i}^{\mathrm{on}} \cdot P_{i}^{\mathrm{min}}+\sum_{\substack{b \in B \\
b>1}} \Delta P_{t, i, b}^{\mathrm{up}} \quad \forall t \in T, \forall i \in \mathrm{UG}
\end{aligned}
$$

(d) Reactive power output limits:

$$
\begin{aligned}
& u_{t, i}^{\text {on }} \cdot Q_{i}^{\min } \leq Q_{t, i}^{c} \leq u_{t, i}^{\text {on }} \cdot Q_{i}^{\max } \quad \forall c \in C, \forall t \in T, \forall i \in \mathrm{UG} \\
& \left(1-u_{t, i}^{\text {off }}\right) \cdot Q_{i}^{\text {min }} \leq Q_{t, i}^{c} \leq\left(1-u_{t, i}^{\text {off }}\right) \cdot Q_{i}^{\max } \quad \forall c \in C, \forall t \in T, \forall i \in \mathrm{CG}
\end{aligned}
$$

(e) Reactive power output by shunt reactors or capacitors:

$$
Q_{t, k}^{c}=B_{k, n}^{\mathrm{sh}} \cdot u_{t, k}^{\mathrm{on}} \cdot\left(V_{t, n}^{c}\right)^{2} \quad \forall c \in C, \forall t \in T, \forall k \in \mathrm{RC}, n \in N: B_{k, n}^{\mathrm{sh}} \neq 0
$$

(f) System operating reserve requirements:

$$
\begin{aligned}
& \sum_{i \in \mathrm{UG}} u_{t, i}^{\mathrm{on}} \cdot P_{i}^{\mathrm{max}}+\sum_{i \in \mathrm{CG}}\left(1-u_{t, i}^{\mathrm{off}}\right) \cdot P_{i}^{\mathrm{max}} \geq \sum_{n \in N} P_{t, n}^{d}+R_{t} \quad \forall t \in T \\
& \sum_{k \in \mathrm{UG}} u_{t, k}^{\mathrm{on}} \cdot Q_{k}^{\mathrm{max}}+\sum_{i \in \mathrm{CG}}\left(1-u_{t, i}^{\mathrm{off}}\right) \cdot Q_{i}^{\mathrm{max}} \geq \sum_{n \in N} Q_{t, n}^{d} \quad \forall t \in T
\end{aligned}
$$

(g) Minimum starting up times:

$$
\begin{array}{ll}
\left(x_{t-1, i}-\mathrm{UT}_{i}\right) \cdot\left(u_{t-1, i}^{\text {on }}-u_{t, i}^{\text {on }}\right) \geq 0 & \forall t \in T, \forall i \in \mathrm{UG} \\
\left(x_{t-1, i}-\mathrm{UT}_{i}\right) \cdot\left(u_{t-1, i}^{\text {off }}-u_{t, i}^{\text {off }}\right) \leq 0 & \forall t \in T, \forall i \in \mathrm{CG}
\end{array}
$$

(h) Minimum starting down times:

$$
\begin{array}{ll}
\left(x_{t-1, i}+\mathrm{DT}_{i}\right) \cdot\left(u_{t, i}^{\mathrm{on}}-u_{t-1, i}^{\mathrm{on}}\right) \leq 0 & \forall t \in T, \forall i \in \mathrm{UG} \\
\left(x_{t-1, i}+\mathrm{DT}_{i}\right) \cdot\left(u_{t, i}^{\text {off }}-u_{t-1, i}^{\text {off }}\right) \geq 0 & \forall t \in T, \forall i \in \mathrm{CG}
\end{array}
$$

(i) Real power flow equations:

$$
\sum_{n \in N} V_{t, j}^{c} \cdot V_{t, n}^{c} \cdot\left(G_{j n}^{c} \cdot \cos \left(\delta_{t, j}^{c}-\delta_{t, n}^{c}\right)+B_{j n}^{c} \cdot \sin \left(\delta_{t, j}^{c}-\delta_{t, n}^{c}\right)\right)=P_{t, j}^{g}-P_{t, j}^{d} \quad \forall c \in C, \forall t \in T, \forall j \in N: P_{t, j}^{g}=\sum_{i \in \Psi_{j}} P_{t, i}
$$

(j) Reactive power flow equations:

$$
\sum_{n \in N} V_{t, j}^{c} \cdot V_{t, n}^{c} \cdot\left(G_{j n}^{c} \cdot \sin \left(\delta_{t, j}^{c}-\delta_{t, n}^{c}\right)-B_{j n}^{c} \cdot \cos \left(\delta_{t, j}^{c}-\delta_{t, n}^{c}\right)\right)=Q_{t, j}^{g, c}-Q_{t, j}^{d} \quad \forall c \in C, \forall t \in T, \forall j \in N: Q_{t, j}^{g, c}=\sum_{k \in \Psi_{j}} Q_{t, k}^{c}
$$

(k) Transmission capacity limits:

$$
\left|V_{t, j}^{c} \cdot\left[\left(V_{t, j}^{c}-V_{t, n}^{c}\right) \cdot y_{j n}\right]^{*}+V_{t, j}^{c} \cdot\left(V_{t, j}^{c} \cdot\left(\frac{1}{2} y_{j n}^{\prime}\right)^{*}\right)\right| \leq S_{j n}^{\max } \forall c \in C, \forall t \in T, \forall j, n \in N:(j n) \in \Phi_{j}
$$


(1) Bus voltage magnitude limits:

$$
V^{\min } \leq V_{t, n}^{c} \leq V^{\max } \quad \forall c \in C, \forall t \in T, \forall n \in N
$$

(m) Pre and post-contingency bus angle limits:

$$
\begin{aligned}
& -\pi \leq \delta_{t, n}^{c} \leq \pi \quad \forall c \in C, \forall t \in T, \forall n \in N /\left\{n_{s}\right\} \\
& \delta_{t, j}^{c}=0 \quad n_{s}: \text { swing bus }
\end{aligned}
$$

(n) Transformer tap limits:

$$
\mathrm{RT}_{n j}^{\min } \leq r t_{t, n j} \leq \mathrm{RT}_{n j}^{\max } \quad \forall t \in T, \forall n, j \in N:(n j) \in \Phi_{n}^{\mathrm{RT}}
$$

(o) Real power output of units after generator outage:

$$
\begin{aligned}
& P_{t, k}^{c}=P_{t, k}+\frac{P_{k}^{\max }-P_{t, k}}{\sum_{k \neq i}\left(P_{k}^{\max }-P_{t, k}\right)} \cdot P_{t, i} \quad c \neq c 0, \forall k \in G: P_{t, k}>0(i \neq k) \\
& P_{t, i}^{c}=0 \quad c \neq c 0, i \in G: P_{t, i}>0 \quad(i \neq k)
\end{aligned}
$$

The $n-1$ security constraints (operating states ' $c$ ': $c \neq c 0$ ) are additional equality and inequality constraints associated to predefined outages (contingency analysis). Each selected outage is characterized by a new set of nodal power flow equations and transmission system operating limits ( $n-1$ state equations), in which the control variables (generator real power, transformer taps and generator voltage magnitude) are kept in equal value that in the base-case (operating state $c 0$ ). The exception is the generating units outages, in which the lost generation will be supplied by the rest of the committed generating units (all of them or a subset, simulating the action of the primary $P-f$ regulation) according to the criterion defined in (23). It should be noted that $V^{\mathrm{min}}, V^{\mathrm{max}}$, $S_{j n}^{\max }$ will be not the same in a normal or post-contingency state.

For any operating state, $G_{j n}^{c}$ and $B_{j n}^{c}$ equals $G_{j n}^{c 0}$ and $B_{j n}^{c 0}$, respectively, except for those elements $j n$ of bus admittance matrix in which the reactor, capacitor, line or transformer whose outage produces out of limit variables are involved.

The constraints (15) and (16) are linearized according to the formulation used in reference [25].

\section{Benders decomposition}

The power scheduling problem addressed in this paper is a mixed-integer non-linear optimization problem with linear objective function, binary decision variables, continuous variables for operation processes, time couplings and non-linear constraints, such as complete power flow equations or pre and post-contingency transmission capacity limits.

The difficulties related to resolution of non-linear optimization problems with binary variables force to make use of partitioning techniques as Benders decomposition [1,2]. In this work, this method is applied to the second stage: technical constraints solution process. The Benders partition algorithm is a decomposition technique in two-levels, master and slave, which defines an iterative procedure between both levels in order to reach the optimal solution. The master level represents the decision problem, mixed-integer linear optimization problem, whereas the slave level deals with the operation problem, multi-period non-linear SCOPF. In this case, the time coupling constraints included in the multi-period subproblem, ramp rate limits (7), are arranged as hourly power limits [25] that are updated after solving each hourly slave subproblem so that production limits of each generator are fulfilled within the next hourly slave subproblem. Consequently, the slave problem may be decomposed in 24 subproblems (one per hour), which are sequentially solved.

The master problem determines the new committed/de-committed generating units, reactors and capacitors. This schedule is transferred to the slave subproblem, which calculates the operating cost and the dual values associated to the scheduling decision taken by the master problem. This information is supplied in the next iteration to the master problem through the Benders cuts to improve the new decision of the master problem.

Therefore, this method allows to treat the non-convexity associated to binary variables and to divide the global problem into two smaller problems easier to solve. The algorithm optimizes jointly the time-coupled daily problem, providing better results than those obtained in case of solving the constraints separately hour by hour, as the SO has usually carried out.

The procedure followed in this paper includes the steps illustrated in the flowchart of Fig. 1. The "start" point will be the result of the daily clearing market.

\subsection{Master problem}

The master problem decides the commitment of new generating units, reactors or capacitors, as well as de-commitment of some generators previously connected at the clearing market. Therefore, all binary variables have to be included in the optimization problem of this level. 


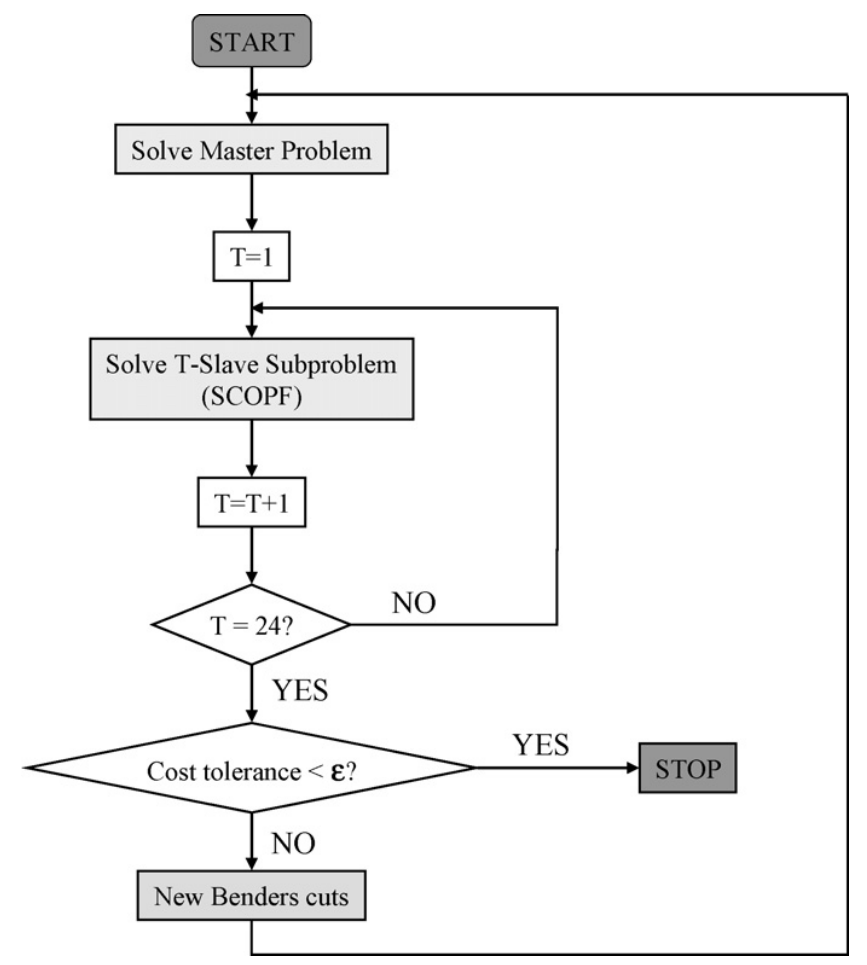

Fig. 1. Flowchart of the decomposition procedure.

The objective function minimizes:

$$
\sum_{t \in T i \in \mathrm{UG}} \sum_{t, i, 1}^{\bullet} \cdot u_{t, i}^{\mathrm{on}} \cdot P_{i}^{\mathrm{min}}+\sum_{t \in T} \alpha_{t}^{*}
$$

subject to the constraints (14)-(16) and:

$$
\alpha_{t}^{*} \geq \alpha_{t}\left(U_{t, k}^{m-1}\right)+\sum_{k \in \mathrm{UG}} \lambda_{t, k}^{m-1} \cdot\left(u_{t, k}^{\mathrm{on}}-U_{t, k}^{m-1}\right)+\sum_{i \in \mathrm{CG}} \lambda_{t, i}^{m-1} \cdot\left(u_{t, i}^{\mathrm{off}}-U_{t, i}^{m-1}\right) \quad \forall t \in T, \forall m \in M
$$

The first term of the objective function represents the "start-up" cost of the unit $i$ at its minimum power (first bid block). The second term means, through the real variable $\alpha_{t}^{*}$, an underestimation of the operating costs of each hourly slave subproblem. Therefore, the optimization variables of this problem are $\alpha_{t}^{*}, u_{t, k}^{\text {on }}, u_{t, i}^{\text {off }}$.

The key issue in Benders decomposition is located at Eq. (25), the named Benders linear cuts. The levels, master and slave, are coupled by these cuts which are updated at each iteration for all operation problems, that is to say, "24" new cuts are added to the master problem at each iteration.

\subsection{Slave subproblem}

The slave level solves the operation problem by means of an AC SCOPF. The daily scheduling is coupled between hours by the ramp rate limits, which are formulated as hourly power limits, so that this slave level can be decomposed in 24 subproblems easier to solve.

Each hourly slave subproblem solves system operation, minimizing the cost of the production bids submitted by each committed generating unit at each hour (except for the first bid block, which has been included in the master problem), starting from the outcome of the daily market. The objective function minimizes at each period:

$$
\sum_{i \in \mathrm{UG}} \sum_{\substack{b \in B \\ b>1}} p_{t, i, b}^{\bullet} \cdot \Delta P_{t, i, b}^{\mathrm{up}}+\sum_{i \in \mathrm{CG} b \in B} \sum_{\substack{b>1 \\ b>1}} p_{t, i, b}^{\bullet} \cdot \Delta P_{t, i, b}^{\mathrm{up}} \quad t \in T
$$


subject to the constraints (7), (9)-(13), (17)-(23) and:

$$
\begin{aligned}
& u_{t, k}^{\mathrm{on}}=U_{t, k}^{m}: \lambda_{t, k}^{m+1} \quad t \in T, \forall k \in \mathrm{UG} \\
& u_{t, i}^{\text {off }}=U_{t, i}^{m}: \lambda_{t, i}^{m+1} \quad t \in T, \forall i \in \mathrm{CG}
\end{aligned}
$$

The minimization is subject to the AC power flow equations ( $n$ and $n-1$ states), real and reactive output production limits, transmission capacity limits of lines, voltage magnitude and bus angle limits, .... Besides, Eq. (27) supplies the sensitivity for each value of the decision variables $\left(u_{t, k}^{\text {on }}, u_{t, i}^{\text {off }}\right)$ fixed by the master problem at the same iteration.

Therefore, the SCOPF is solved adding the constraints of the previously selected non-secure $(n-1)$ contingency cases to model the system limits during post-contingency state [26]. Each slave problem determines the values of the operation variables $\left(P_{t, i}, Q_{t, k}\right.$, $\left.V_{t, n}, Q_{t, k}^{c}, V_{t, n}^{c}, \delta_{t, n}^{c}, r_{t, n j}\right)$ at each period for all operating states.

\subsection{Benders convergence criterion}

Benders decomposition procedure stops when the value of the objective function computed in the slave problem (operating costs) plus 'start-up' costs $\left(\sum_{t \in T} \sum_{i \in \mathrm{UG}} p_{t, i, 1}^{\bullet} \cdot u_{t, i}^{\text {on }} \cdot P_{i}^{\mathrm{min}}\right)$ equals master problem cost, except for a small cost tolerance: $\varepsilon$. Actually, as the start-up costs are the same in both slave and master problems, they can be omitted in the convergence criterion (CC), considering only the operating cost in slave and master problems. The final convergence criterion is established as Eq. (28) shows.

$$
\mathrm{CC}=\frac{\sum_{t \in T}\left(\alpha_{t}\left(U_{t, i}^{m}\right)-\alpha_{t}^{*}\right)}{\sum_{t \in T} \alpha_{t}\left(U_{t, i}^{m}\right)} \leq \varepsilon \quad \forall m \in M
$$

\subsection{Slave problems feasibility}

Feasibility cuts have been added to the master problem to enforce the feasibility of the slave problems. However, since voltage control is a local problem, there could be some cases where the reactive power constraint (31) does not guarantee the problem feasibility. It would force to add fictitious sources at some PV buses or at buses with voltage control elements and to include them with a penalty factor in the objective function of the slave problem (26) so that the new objective function optimizes the global cost minimizing infeasibilities costs as well. The objective function would be formulated as:

$$
\begin{gathered}
\sum_{i \in \mathrm{UG}} \sum_{\substack{b \in B \\
b>1}} p_{t, i, b}^{\bullet} \cdot \Delta P_{t, i, b}^{\mathrm{up}}+\sum_{\substack{i \in \mathrm{CG} \\
b \in B}} \sum_{b>1} p_{t, i, b}^{\bullet} \cdot \Delta P_{t, i, b}^{\mathrm{up}}+\sum_{n \in N} \mathrm{Cp} \cdot\left(P_{t, n}^{\mathrm{fic}}+Q_{t, n}^{\mathrm{fic} L}+Q_{t, n}^{\mathrm{fic} C}\right) \quad t \in T \\
b>1
\end{gathered}
$$

These new cuts replace Eq. (14) and improve the problem convergence, reducing the number of iterations. They are formulated as:

$$
\begin{aligned}
& \sum_{i \in \mathrm{UG}} u_{t, i}^{\mathrm{on}} \cdot P_{t, i}^{\mathrm{max}}+\sum_{i \in \mathrm{CG}}\left(1-u_{t, i}^{\mathrm{off}}\right) \cdot P_{t, i}^{\mathrm{max}} \geq \sum_{n \in N} P_{t, n}^{d}+R_{t} \quad \forall t \in T \\
& \sum_{i \in \mathrm{UG}} u_{t, i}^{\mathrm{on}} \cdot P_{t, i}^{\mathrm{min}}+\sum_{i \in \mathrm{CG}}\left(1-u_{t, i}^{\mathrm{off}}\right) \cdot P_{t, i}^{\mathrm{min}} \leq \sum_{n \in N} P_{t, n}^{d} \quad \forall t \in T \\
& \sum_{k \in \mathrm{UG}} u_{t, k}^{\mathrm{on}} \cdot Q_{k}^{\max }+\sum_{i \in \mathrm{CG}}\left(1-u_{t, i}^{\mathrm{off}}\right) \cdot Q_{i}^{\max } \geq \sum_{n \in N} Q_{t, n}^{d} \quad \forall t \in T \\
& \sum_{k \in \mathrm{UG}} u_{t, k}^{\mathrm{on}} \cdot Q_{k}^{\mathrm{min}}+\sum_{i \in \mathrm{CG}}\left(1-u_{t, i}^{\mathrm{off}}\right) \cdot Q_{i}^{\mathrm{min}} \leq \sum_{n \in N} Q_{t, n}^{d} \quad \forall t \in T
\end{aligned}
$$

\section{Test systems and results}

The test systems are the IEEE 24-bus Reliability Test System and an adapted IEEE 118-bus Test System with standard costs for the generating units. Only results for the IEEE 24-bus system are shown. The test carried out for the IEEE 118-bus system seeks to compare the behaviour of the proposed approach in a larger power system with an important number of voltage control devices (12 capacitors and 2 reactors) and to compare the execution times.

The IEEE 24-bus Reliability Test System includes 32 units: nuclear, coal, oil and hydro plants, distributed all over the generating buses and ranging from 12 to $400 \mathrm{MW}$. The total generation capacity amounts to $3405 \mathrm{MW}$. The transmission network contains 24 load/generating buses connected by 38 AC lines or transformers at two voltages, 138 and $230 \mathrm{kV}$. The swing bus is N13 and it is assumed that one of the generating units located at this bus is always connected. 


\begin{tabular}{|c|c|c|}
\hline Hours $(t)$ & Lines or transformers $(j n)$ & Generating units $(i)$ \\
\hline$\{\mathrm{T} 7<t<\mathrm{T} 22\}$ & $(\mathrm{N} 7-\mathrm{N} 8)$ & \\
\hline$t=\{\mathrm{T} 3, \mathrm{~T} 6\}$ & (N7-N8), (N8-N9) & G9 or G10 or G11 at bus N7 \\
\hline$t=\{\mathrm{T} 1, \mathrm{~T} 2, \mathrm{~T} 4, \mathrm{~T} 5, \mathrm{~T} 22, \mathrm{~T} 24\}$ & $(\mathrm{N} 7-\mathrm{N} 8),(\mathrm{N} 8-\mathrm{N} 9),(\mathrm{N} 8-\mathrm{N} 10)$ & G9 or G10 or G11 \\
\hline$t=\{\mathrm{T} 23\}$ & $(\mathrm{N} 7-\mathrm{N} 8),(\mathrm{N} 8-\mathrm{N} 9),(\mathrm{N} 8-\mathrm{N} 10),(\mathrm{N} 3-\mathrm{N} 24),(\mathrm{N} 9-\mathrm{N} 11)$ & G9 or $\mathrm{G} 10$ or $\mathrm{G} 11$ \\
\hline$t=\{\mathrm{T} 7\}$ & (N7-N8), (N8-N9), (N8-N10), (N3-N24), (N9-N11), (N11-N14), (N12-N23), (N15-N24) & G9 or $\mathrm{G} 10$ or $\mathrm{G} 11$ \\
\hline
\end{tabular}

The power system has voltage control devices at buses N14 (synchronous condenser) and N6 (reactor). The transformers taps are used as voltage control variables and they are modelled as continuous variables. The voltage limits in n-state are 0.95 and 1.05 . In a post-fault state, the limits are set to 0.93 and 1.11. The flow limits are provided in reference [20].

A 24-h time horizon is considered. The peak load for the test system is $2850 \mathrm{MW}$ and occurs in 18 and $19 \mathrm{~h}$. The minimum load is $1682 \mathrm{MW}$ and takes place in 4 and $5 \mathrm{~h}$. A $98 \%$ power factor is applied to all load levels.

The power production system is organized as a market based on prices. The generators bid prices have been taken according to the marginal costs of each energy bid block and rise with the block number. The features of the generating units, the energy offer blocks and their bid prices and the layout of IEEE 24-bus Test System are shown in Appendix A.

After daily market, some severe contingencies are selected (see Table 1). The electric problems caused by these contingencies are:

- The lost of the line (N7-N8) for the periods $\{\mathrm{T} 1 / \mathrm{T} 7\}$ and T24, where the solution provided by the daily market does not connect any generator at bus N7, implies that the bus N7 demand is not supplied. In the rest of periods, the lost of this line provokes the bus N7 is left cut off, working as an electric island, being critical the connection of the units located at this bus (G9, G10 or G11). The constraints solution will set, at least, one generator at bus N7.

- The lost of the line (N8-N9) involves low-voltage problems in the buses N7 and N9, which spread over the bus N8 in certain periods. The commitments of units at bus N7 and at the swing bus N13, as well as, the synchronous condenser at bus N14 solve this low-voltage problem.

- The contingency (N8-N10) implies low-voltage problems in the bus N7, and also in bus N8 for some periods. The commitment of units at bus N7 and at the swing bus N13 improves this voltages outline.

- The lost of the line (N3-N24) results in low-voltage problems in the bus N7 and over-voltage problems in the bus N6. This contingency can be solved by the commitment of the synchronous condenser at bus N14.

- The rest of contingencies entail voltage problems in the network buses.

The Spanish SO usually solves technical constraints process hour by hour, but it does not guarantee the optimal solution. In this section, the IEEE test system is used to justify the need of a full time-coupled SCOPF model in order to solve the secure daily generation scheduling problem in an optimal way. For this purpose, two study cases are compared:

- Case I: Daily market followed by constraints solution procedure, which is solved hour by hour (contingency analysis + hourly AC SCOPF: rescheduling). In this case, both master and slave problems would be hourly optimization problem.

- Case II: Daily market followed by time-coupled constraints solution procedure (complete multi-period AC SCOPF model).

The obtained solutions for both cases use the same resources, generators at N7 and N13 and synchronous condenser, to solve constraints, but the solutions differ in the start-ups number of these units needed to provide a secure scheduling.

The three generating units located at bus N7 (G9, G10 and G11) are going to be used to compare the solution obtained in both cases. The previous status (on/off) of each generator is supplied by the solution of the daily market. In the daily market, no generator at bus N7 is connected during the first seven periods, T23 and T24, while from T9 to T21, the three units are committed.

On the one hand, the time-decoupled case (case I) would only minimize, separately each period, the redispatching cost and, therefore, the master problem would not include constraints (15) and (16). On the other hand, the case II minimizes throughout the day both the redispatching cost and the number of start-ups. In Figs. 2 and 3, it is shown the difference between the optimization of both cases referred to these units G9, G10 and G11. As it can be noticed in Fig. 3, it is committed an only unit (G9) during the first seven periods unlike the case I, where the three generators have been committed. Besides, it is not necessary the start-up of this unit, as it was committed in the last hour of the day before: $U_{0, \mathrm{G} 9}=1$. For the last programming periods, T22-T24, the scheduling in both cases is quite different. In the case II, the three generating units are committed, but none of them have been started up to solve constraints, as they were already committed in the daily market for the previous periods. In the rest of periods, the differences are only because of the supplied solution by the optimizer. 


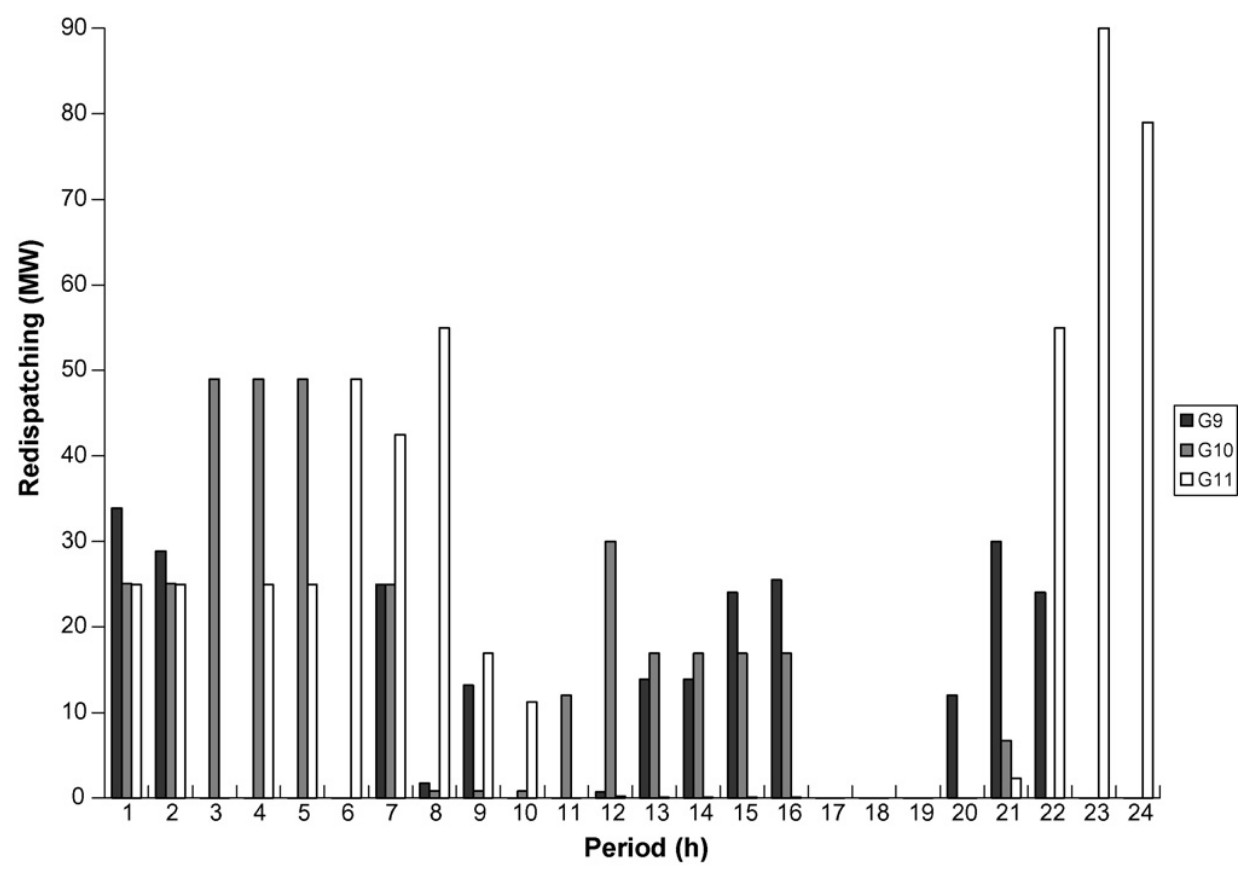

Fig. 2. Case I: redispatching of the generating units located at bus N7.

Therefore, Figs. 2 and 3 show the differences between both scheduling cases related to units G9, G10 and G11. These results indicate that, in the case II, there are two only start-ups (units G10 and G11) at periods T7 and T8, respectively, while in the case I there are seven start-ups in all for the three units throughout day. In the study model (based on bid prices regarding from marginal costs), the start-up or shut-down costs are not included, and there are no significant differences in the final costs provided by both solutions. But, if the start-up cost was included in the bids, it would imply an important over-cost for the case I. For example, on the assumption of US\$ 600 as hot start-up cost of units located at N7 [20], it will entail an over-cost of US\$ 3000 per day. Consequently, the case I (present Spanish model) does not supply the optimal solution of the secure daily power scheduling problem.

The general model, case II, manages in the master problem 816 binary variables, 624 integer variables and 24 continuous variables. Each hourly slave problem includes from 357 variables and 644 constraints on, depending on the number of contingencies. Each contingency adds 227 new constraints and 77 new variables. The master problem is solved using CPLEX [24] under GAMS, whereas the slave subproblem is solved using CONOPT [23]. The per unit cost tolerance $\varepsilon$ is fixed to $1 \mathrm{e}-3$.

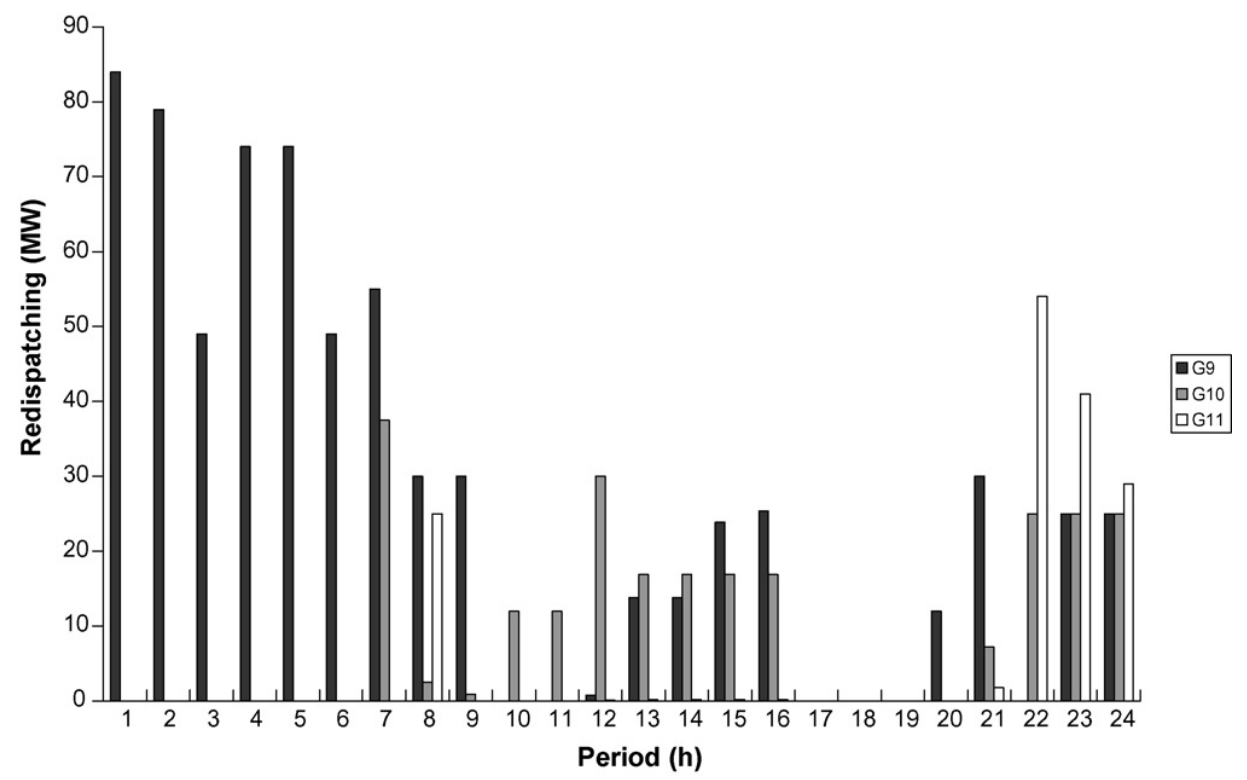

Fig. 3. Case II: redispatching of the generating units located at bus N7. 
Evolution of the convergence of costs (US\$) with the number of iterations

\begin{tabular}{|c|c|c|c|c|}
\hline Iteration & Master total cost & Slave operating cost & Master operating cost & $\mathrm{CC}$ \\
\hline 2 & 29751.627 & 765802.653 & 11392.014 & 0.985 \\
\hline 3 & 37020.151 & 94600.365 & 14947.913 & 0.842 \\
\hline 4 & 39585.380 & 20452.575 & 17329.531 & 0.153 \\
\hline 5 & 41193.818 & 18465.134 & 18060.830 & 0.022 \\
\hline 6 & 41598.122 & 18465.134 & 18465.134 & 0.0 \\
\hline
\end{tabular}

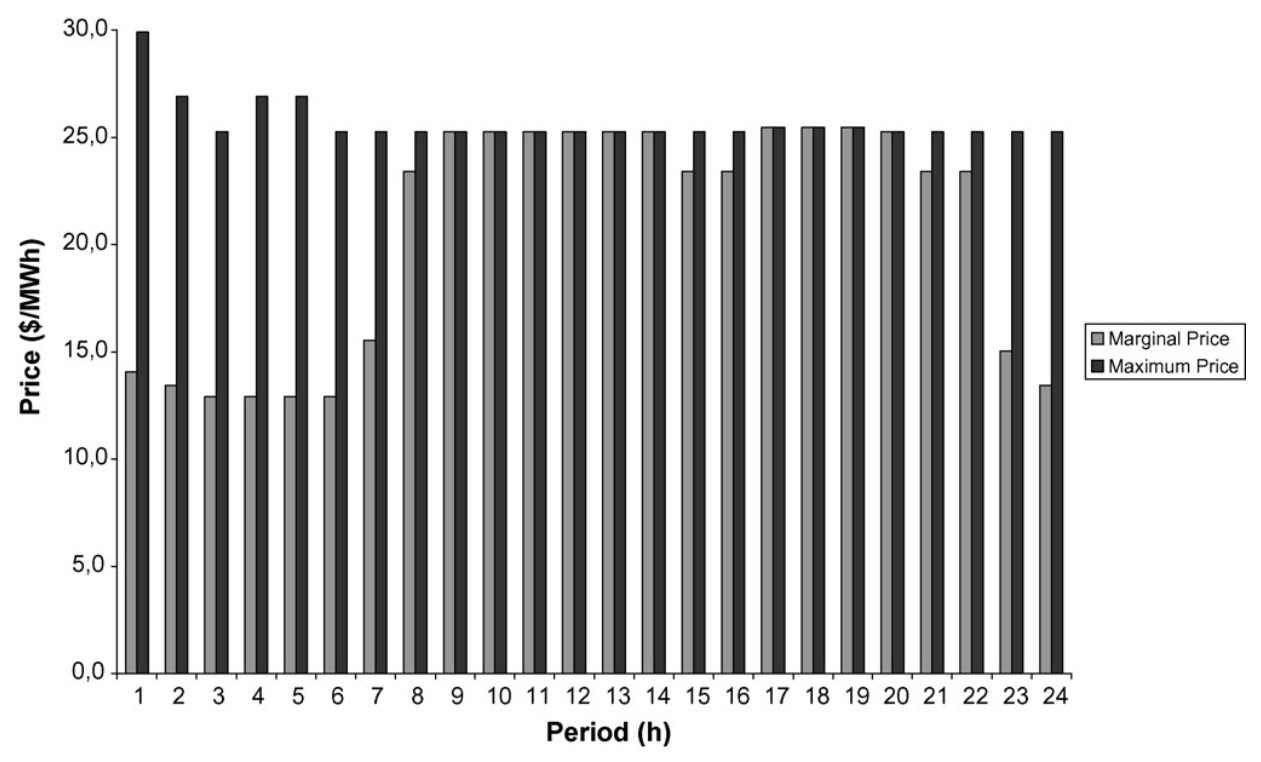

Fig. 4. Hourly marginal price and hourly maximum price.

The most relevant results supplied by the solution are status (on/off) of every generating unit, reactor and capacitor per hour; active power output of every plant per hour; reactive power output of every plant and voltage control devices per hour; total system cost; market clearing price at each hour; voltage magnitude of every bus per hour; power flow of every line per period, .... In relation to optimal power scheduling of the general model (case II), Table 2 shows as the difference between operating costs of the slave and master problems is progressively decreasing with the number of iterations. The master total cost informs of the cost of the technical constraint resolution process.

Fig. 4 shows the system marginal price without constraints (daily market) and the hourly maximum price after constraints solution process. The latter is the highest price paid to a generator included as a consequence of the constraints solution. The market clearing price follows the load curve, whereas the maximum price is determined by the new generating units committed to solve constraints.

The generating units located at buses $\mathrm{N} 7$ and N13, which are more expensive, determine the final price at each period. It may be deduced that these units have a privileged location in the network to solve constraints.

The number of iterations to reach the convergence and the total CPU time required carrying out the study cases on an INTEL P-IV (3.06 GHz, memory: $1 \mathrm{~Gb})$ are shown in Table 3.

The application of this method to real systems needs to be improved in the future in order to reduce computation time [19]. In large power systems, the execution time is highly dependent on the computation efficiency of non-linear optimizer (CONOPT under GAMS is too slow for highly non-linear large systems), the required precision to linear mixed-integer optimizer, the start point to run the hourly SCOPF and the number of contingencies analyzed. Therefore, the computation times could be reduced by using more efficient computation tools, by choosing a best start point based on $\mathrm{SO}$ experience, by parallel programming and/or defining reactive reserve by areas. Heuristic approximation criteria as the realistic reduction of number of binary variables (the most of the units will not be de-committed) would also reduce the problem complexity. Future developments aim at a solution of this problem.

Table 3

Computation time and number of iterations in test systems

\begin{tabular}{llr}
\hline & Computation time & Number of iterations \\
\hline IEEE 24-bus system & 1 min 26s & 6 \\
IEEE 118-bus system & $10 \min 20 \mathrm{~s}$ & 12 \\
\hline
\end{tabular}




\section{Conclusion}

The generalized Benders decomposition algorithm is used to solve a multi-period dispatch problem with security constraints in a pool-organized electricity market model. Specifically, GBD algorithm is applied to solve technical constraints procedure. The study model takes into account some features of the Spanish wholesale market.

The proposed method improves presently available approaches in the following respects: (a) the daily dispatch considers simultaneously dispatch constraints with a precise $\mathrm{AC}$ model of the transmission network including power flow equations, line capacity limits and voltage limits in both pre and post-contingency states; (b) the model provides an optimal and preventive real power schedule with voltages control devices commitment and solves jointly overloads and voltage constraints; (c) the algorithm optimizes jointly the 24-h problem (unit commitment with SCOPF) providing better results than those obtained solving each hourly problem separately; (d) the algorithm is effective, flexible (adaptable to different study models) and decomposable (it allows the future implementation of parallel programming to solve each hourly slave subproblem).

A small-scale case study based on the IEEE 24-bus is analyzed and the computation time and number of iterations are compared with the IEEE 118-bus System. The method shows good convergence properties for the developed application.

Concerning the results, the hourly maximum price allows knowing the new generating units committed to solve constraints. In the same way, the evolution of the marginal prices reports on the load level and the marginal power plant for each period.

\section{Appendix A}

The features of the generating units are shown in Table A.1, assuming a base power of $100 \mathrm{MVA}$ and being $U_{i}^{0}$ and $D_{i}^{0}$ the number of periods the unit has been on/off-line, respectively, at the beginning of the market horizon. The ramp-up ( $\left.\mathrm{UR}_{i}\right)$ or ramp-down $\left(\mathrm{DR}_{i}\right)$ rate are considered the same value for each generator (see Fig. A1).

The energy offer blocks and their bid prices are shown in Table A.2.

Table A.1

Features of the generating units

\begin{tabular}{|c|c|c|c|c|c|c|c|c|c|c|c|}
\hline Unit & Bus & $P_{i}^{\min }(\mathrm{pu})$ & $P_{i}^{\max }(\mathrm{pu})$ & $Q_{i}^{\min }(\mathrm{pu})$ & $Q_{i}^{\max }(\mathrm{pu})$ & $\mathrm{DT}_{i}(\mathrm{~h})$ & $\mathrm{UT}_{i}(\mathrm{~h})$ & $\mathrm{UR}_{i}(\mathrm{MW} / \mathrm{min})$ & $U_{i}^{0}(\mathrm{~h})$ & $D_{i}^{0}(\mathrm{~h})$ & $P_{i}^{0}(\mathrm{pu})$ \\
\hline G1 & N1 & 0.158 & 0.2 & 0 & 0.10 & 1 & 1 & 3 & 0 & 12 & 0 \\
\hline $\mathrm{G} 2$ & N1 & 0.158 & 0.2 & 0 & 0.10 & 1 & 1 & 3 & 0 & 12 & 0 \\
\hline G3 & N1 & 0.152 & 0.76 & -0.25 & 0.3 & 4 & 8 & 2 & 10 & 0 & 0.38 \\
\hline G4 & N1 & 0.152 & 0.76 & -0.25 & 0.3 & 4 & 8 & 2 & 10 & 0 & 0.38 \\
\hline G5 & $\mathrm{N} 2$ & 0.158 & 0.2 & 0 & 0.10 & 1 & 1 & 3 & 0 & 12 & 0 \\
\hline G6 & $\mathrm{N} 2$ & 0.158 & 0.2 & 0 & 0.10 & 1 & 1 & 3 & 0 & 12 & 0 \\
\hline G7 & $\mathrm{N} 2$ & 0.152 & 0.76 & -0.25 & 0.3 & 4 & 8 & 2 & 10 & 0 & 0.38 \\
\hline G8 & $\mathrm{N} 2$ & 0.152 & 0.76 & -0.25 & 0.3 & 4 & 8 & 2 & 10 & 0 & 0.38 \\
\hline G9 & N7 & 0.25 & 1.0 & 0 & 0.6 & 8 & 8 & 7 & 6 & 0 & 0.25 \\
\hline G10 & N7 & 0.25 & 1.0 & 0 & 0.6 & 8 & 8 & 7 & 0 & 5 & 0 \\
\hline G11 & N7 & 0.25 & 1.0 & 0 & 0.6 & 8 & 8 & 7 & 0 & 3 & 0 \\
\hline G12 & N13 & 0.69 & 1.97 & 0 & 0.8 & 10 & 12 & 3 & 14 & 0 & 0.69 \\
\hline G13 & N13 & 0.69 & 1.97 & 0 & 0.8 & 10 & 12 & 3 & 0 & 8 & 0 \\
\hline G14 & N13 & 0.69 & 1.97 & 0 & 0.8 & 10 & 12 & 3 & 0 & 3 & 0 \\
\hline G15 & N15 & 0.024 & 0.12 & 0 & 0.06 & 2 & 4 & 1 & 0 & 4 & 0 \\
\hline G16 & N15 & 0.024 & 0.12 & 0 & 0.06 & 2 & 4 & 1 & 0 & 4 & 0 \\
\hline G17 & N15 & 0.024 & 0.12 & 0 & 0.06 & 2 & 4 & 1 & 0 & 4 & 0 \\
\hline G18 & N15 & 0.024 & 0.12 & 0 & 0.06 & 2 & 4 & 1 & 0 & 4 & 0 \\
\hline G19 & N15 & 0.024 & 0.12 & 0 & 0.06 & 2 & 4 & 1 & 0 & 4 & 0 \\
\hline G20 & N15 & 0.542 & 1.55 & -0.5 & 0.8 & 8 & 8 & 3 & 12 & 0 & 1.24 \\
\hline G21 & N16 & 0.542 & 1.55 & -0.5 & 0.8 & 8 & 8 & 3 & 12 & 0 & 1.55 \\
\hline G22 & N18 & 1.00 & 4.00 & -0.5 & 2.0 & 1 & 1 & 20 & 12 & 0 & 4.0 \\
\hline G23 & N21 & 1.00 & 4.00 & -0.5 & 2.0 & 1 & 1 & 20 & 12 & 0 & 4.0 \\
\hline G24 & N22 & 0 & 0.5 & -0.1 & 0.16 & 0 & 0 & 0 & 10 & 0 & 0.5 \\
\hline G25 & N22 & 0 & 0.5 & -0.1 & 0.16 & 0 & 0 & 0 & 10 & 0 & 0.5 \\
\hline G26 & N22 & 0 & 0.5 & -0.1 & 0.16 & 0 & 0 & 0 & 10 & 0 & 0.5 \\
\hline G27 & N22 & 0 & 0.5 & -0.1 & 0.16 & 0 & 0 & 0 & 10 & 0 & 0.5 \\
\hline G28 & N22 & 0 & 0.5 & -0.1 & 0.16 & 0 & 0 & 0 & 10 & 0 & 0.5 \\
\hline G29 & N22 & 0 & 0.5 & -0.1 & 0.16 & 0 & 0 & 0 & 10 & 0 & 0.5 \\
\hline G30 & N23 & 0.542 & 1.55 & -0.5 & 0.8 & 8 & 8 & 3 & 12 & 0 & 1.24 \\
\hline G31 & N23 & 0.542 & 1.55 & -0.5 & 0.8 & 8 & 8 & 3 & 12 & 0 & 1.55 \\
\hline G32 & N23 & 1.4 & 3.5 & -0.25 & 1.5 & 48 & 24 & 4 & 24 & 0 & 2.8 \\
\hline
\end{tabular}




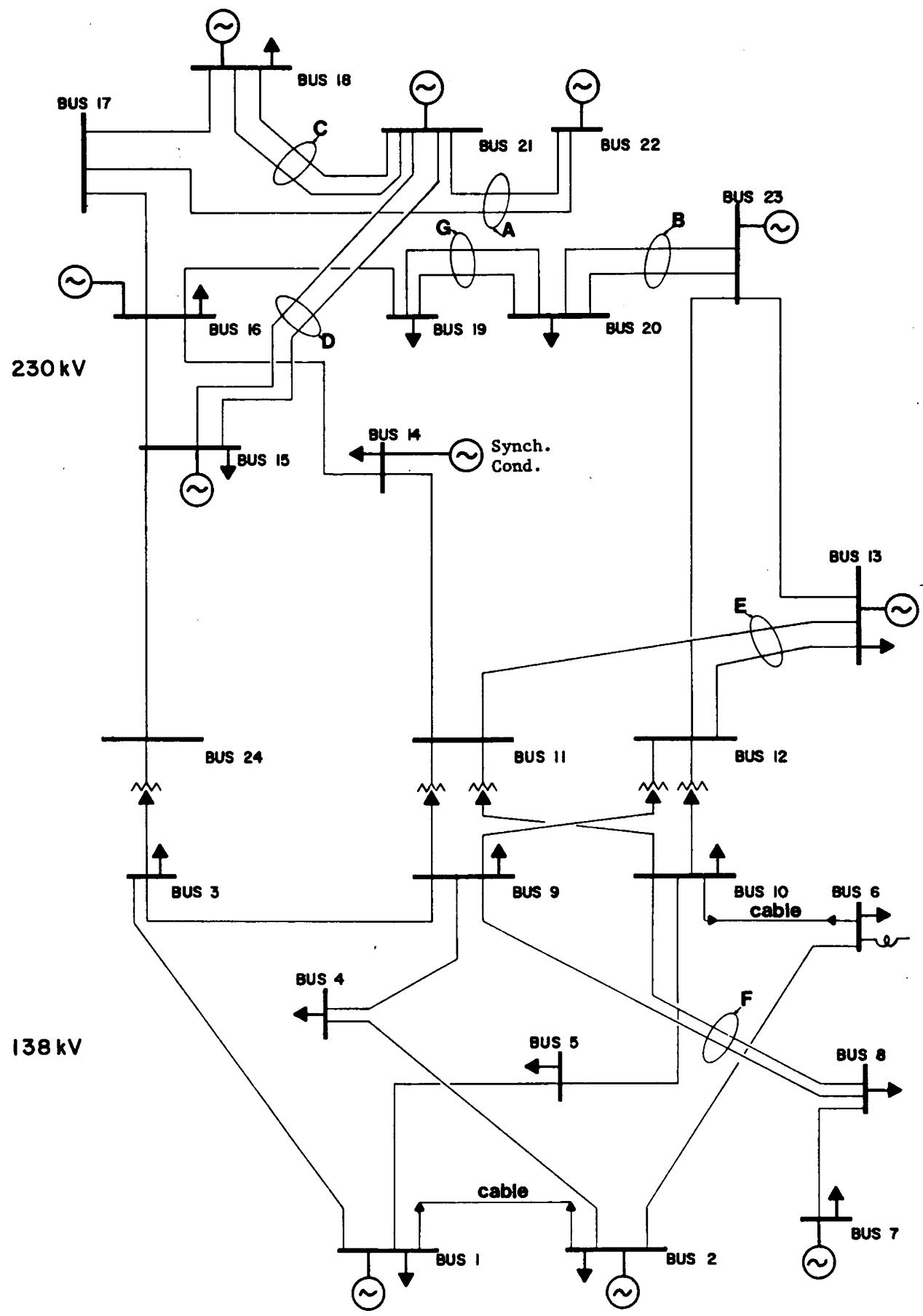

Fig. A1. Layout of IEEE 24-bus test system. 
Table A.2

Energy multi-block price bids of the generating units

\begin{tabular}{|c|c|c|c|c|c|c|c|c|}
\hline Unit & $P_{t, i, 1}(\mathrm{pu})$ & $P_{t, i, 2}(\mathrm{pu})$ & $P_{t, i, 3}(\mathrm{pu})$ & $P_{t, i, 4}(\mathrm{pu})$ & $p_{t, i, 1}^{\bullet}(\mathrm{US} \$ / \mathrm{MWh})$ & $p_{t, i, 2}^{\bullet}(\mathrm{US} \$ / \mathrm{MWh})$ & $p_{t, i, 3}^{\bullet}(\mathrm{US} \$ / \mathrm{MWh})$ & $p_{t, i, 4}^{\bullet}(\mathrm{US} \$ / \mathrm{MWh})$ \\
\hline G1 & 0.158 & 0.002 & 0.038 & 0.002 & 45.189 & 89.316 & 95.022 & 96.777 \\
\hline $\mathrm{G} 2$ & 0.158 & 0.002 & 0.038 & 0.002 & 45.189 & 89.316 & 95.022 & 96.777 \\
\hline G3 & 0.152 & 0.228 & 0.228 & 0.152 & 12.294 & 14.061 & 18.878 & 25.479 \\
\hline G4 & 0.152 & 0.228 & 0.228 & 0.152 & 12.294 & 14.061 & 18.878 & 25.479 \\
\hline G5 & 0.158 & 0.002 & 0.038 & 0.002 & 45.189 & 89.316 & 95.022 & 96.777 \\
\hline G6 & 0.158 & 0.002 & 0.038 & 0.002 & 45.189 & 89.316 & 95.022 & 96.777 \\
\hline G7 & 0.152 & 0.228 & 0.228 & 0.152 & 12.294 & 14.061 & 18.878 & 25.479 \\
\hline G8 & 0.152 & 0.228 & 0.228 & 0.152 & 12.294 & 14.061 & 18.878 & 25.479 \\
\hline G9 & 0.25 & 0.3 & 0.25 & 0.2 & 21.215 & 25.269 & 26.922 & 29.900 \\
\hline G10 & 0.25 & 0.3 & 0.25 & 0.2 & 21.215 & 25.269 & 26.922 & 29.900 \\
\hline G11 & 0.25 & 0.3 & 0.25 & 0.2 & 21.215 & 25.269 & 26.922 & 29.900 \\
\hline G12 & 0.69 & 0.492 & 0.394 & 0.394 & 21.878 & 23.413 & 25.457 & 27.295 \\
\hline G13 & 0.69 & 0.492 & 0.394 & 0.394 & 21.878 & 23.413 & 25.457 & 27.295 \\
\hline G14 & 0.69 & 0.492 & 0.394 & 0.394 & 21.878 & 23.413 & 25.457 & 27.295 \\
\hline G15 & 0.024 & 0.036 & 0.036 & 0.024 & 23.991 & 26.963 & 34.937 & 47.645 \\
\hline G16 & 0.024 & 0.036 & 0.036 & 0.024 & 23.991 & 26.963 & 34.937 & 47.645 \\
\hline G17 & 0.024 & 0.036 & 0.036 & 0.024 & 23.991 & 26.963 & 34.937 & 47.645 \\
\hline G18 & 0.024 & 0.036 & 0.036 & 0.024 & 23.99 & 26.963 & 34.937 & 47.645 \\
\hline G19 & 0.024 & 0.036 & 0.036 & 0.024 & 23.991 & 26.963 & 34.937 & 47.645 \\
\hline G20 & 0.542 & 0.388 & 0.31 & 0.31 & 10.713 & 12.179 & 13.438 & 15.032 \\
\hline G21 & 0.542 & 0.388 & 0.31 & 0.31 & 10.713 & 12.179 & 13.438 & 15.032 \\
\hline G22 & 1.00 & 1.0 & 1.2 & 0.8 & 5.449 & 5.673 & 5.995 & 6.434 \\
\hline G23 & 1.00 & 1.0 & 1.2 & 0.8 & 5.449 & 5.673 & 5.995 & 6.434 \\
\hline $\mathrm{G} 24$ & 0 & 0.15 & 0.15 & 0.20 & 0 & 0.01 & 0.1 & 0.19 \\
\hline G25 & 0 & 0.15 & 0.15 & 0.20 & 0 & 0.01 & 0.1 & 0.19 \\
\hline G26 & 0 & 0.15 & 0.15 & 0.20 & 0 & 0.01 & 0.1 & 0.19 \\
\hline G27 & 0 & 0.15 & 0.15 & 0.20 & 0 & 0.01 & 0.1 & 0.19 \\
\hline G28 & 0 & 0.15 & 0.15 & 0.20 & 0 & 0.01 & 0.1 & 0.19 \\
\hline G29 & 0 & 0.15 & 0.15 & 0.20 & 0 & 0.01 & 0.1 & 0.19 \\
\hline G30 & 0.542 & 0.388 & 0.31 & 0.31 & 10.713 & 12.179 & 13.438 & 15.032 \\
\hline G31 & 0.542 & 0.388 & 0.31 & 0.31 & 10.713 & 12.179 & 13.438 & 15.032 \\
\hline G32 & 1.4 & 0.875 & 0.525 & 0.7 & 11.623 & 12.918 & 14.196 & 15.531 \\
\hline
\end{tabular}

\section{Appendix B}

\section{B.1. List of symbols}

$B_{j n}^{c} \quad$ imaginary term of the element $j, n$ in bus admittance matrix for the operating state $c$

$B_{k, n}^{\text {sh }} \quad$ susceptance of the capacitor or reactor $k$ connected at bus $n$

$\mathrm{Cp} \quad$ penalization factor associated to the fictitious generating sources

$\mathrm{DR}_{i} \quad$ ramp-down and shut-down rate limit $(\mathrm{MW} / \mathrm{h})$ of unit $I$

$\mathrm{DT}_{i} \quad$ minimum down time of unit $i$

$G_{j n}^{c} \quad$ real term of the element $j, n$ in bus admittance matrix for the operating state $c$

$P_{t, i} \quad$ real power output of generating unit $i$ at period $t$

$P_{t, i, b} \quad$ real power of block $b$ offered by unit $i$ at period $t$

$P_{t, i}^{c} \quad$ real power output of unit $i$ at period $t$ for the operating state $c$ defined by the lost of one generator

$P_{t, j}^{g} \quad$ real power injected by all the generating units connected at bus $j$ at period $t$

$P_{t, n}^{\text {fic }} \quad$ real power injected by fictitious sources connected at bus $n$ for period $t$

$P_{i}^{\max } \quad$ maximum real power output of generation unit $i$

$P_{i}^{\min } \quad$ minimum real power output of generation unit $i$

$P_{t, i}^{\max } \quad$ maximum real power output of generation unit $i$ at period $t$

$P_{t, i}^{\min } \quad$ minimum real power output of generation unit $i$ at period $t$

$P_{t, i, b}^{\max } \quad$ maximum real power offered by the generating unit $i$ for the block $b$ at period $t$

$P_{t, i}^{0} \quad$ real power committed by the generating unit $i$ at period $t$ in the daily market

$P_{t}^{\text {loss }} \quad$ estimation of the system losses for the period $t$ 
Sets

C

CG

G

GR

M

$N$

$N_{c}$

$\mathrm{RC}$

$T$

UG

$\Phi$

$\Phi_{n}$

real load demand at bus $n$ during period $t$

real power of the unit $i$ at period $t$ for iteration $m$

real power increase of block $b$ offered by unit $i$ at period $t$

real power decrease of block $b$ offered by unit $i$ at period $t$

market clearing price at hour $t$

price offered by unit $i$ at hour $t$ for block $b$

reactive power output of generating unit, reactor or capacitor $k$ at period $t$ for the operating state $c$

reactive load demand at bus $n$ during period $t$

reactive power injected by all the units, reactors and capacitors connected at bus $j$ at period $t$ for the operating state $c$

reactive power injected by fictitious sources connected at bus $n$ for period $t$

reactive power absorbed by fictitious sources connected at bus $n$ for period $t$

maximum reactive power output of unit $i$

minimum reactive power output of unit $i$

reserve requirement during period $t$

maximum tap of transformer $(\mathrm{jn})$ at any period $t$

minimum tap of transformer ( $\mathrm{jn}$ ) at any period $t$

continuous variable that represents the tap value for transformer $(n j)$ at period $t$

maximum transmission capacity (MVA) at line (jn) for $n$ state

ramp-up and start-up rate limit (MW/h) of unit $i$

minimum up time of unit $i$

state (on/off) of the unit $i$ at period $t$ for iteration $m$

binary variable (0/1) that represents the commitment state of generating unit $i$ at period $t$ in the daily market

binary decision variable that represents the commitment (0/1) of unit, reactor or capacitor $k$ at period $t$ in the technical constraints solution process

binary decision variable that represents the de-commitment (1/0) of generator $i$ at period $t$ in the technical constraints solution process

bus voltage at bus $n$ during period $t$ for the operating state $c$

bus voltage magnitude at bus $n$ during period $t$ for the operating state $c$

minimum voltage at any node $n$ and any period $t$ for any $(n-1)$ state

maximum voltage at any node $n$ and any period $t$

number of hours that generating unit $i$ has been on (+) or off (-) at the end of hour $t$

line series admittance

charging admittance

phase angle at bus $n$ during period $t$ for the operating state $c$

per unit cost tolerance

dual variable supplied by the slave subproblem in each iteration $m$, which is associated to the decision of connecting/disconnecting of unit $k$ at period $t$

set of indexes of energy sale blocks

set of indexes of all operating states

set of indexes of all committed generating units in the daily market

set of indexes of all generation units

set of indexes of all generation units, reactors and capacitors

set of iteration indexes

set of indexes of all buses

set of all load buses

set of indexes of all reactors and capacitors

set of all period indexes in hours

set of indexes of all reactors, capacitors and all non-committed generators in the daily market

set of all system branches and transformers

subset of all system branches connected at bus $n$

subset of all system transformers connected at bus $n$

subset of indexes of all the generation units at bus $n$ 


\section{References}

[1] J.F. Benders, Partitioning procedures for solving mixed-variables programming problems, Numer. Math. 4 (1962) $238-252$.

[2] A.M. Geoffrion, Generalized Benders decomposition, J. Optim. Theory Appl. (JOTA) 10 (4) (1972) 237-260.

[3] G.B. Sheblé, G.N. Fahd, Unit commitment literature synopsis, IEEE Trans. Power Syst. 9 (1) (1994) 128-135.

[4] Unit Commitment, Task Force 38.04.01, CIGRÉ, August 1998.

[5] Spanish Market Activity Rules (English version), BOE, April 1991, available: http://www.omel.es.

[6] Spanish Ministry of Industry and Energy, Operational Procedures of the Spanish Power System, Madrid, August 1998 (no English version) (online), available: http://www.ree.es.

[7] ETSO, Evaluation of congestion management methods for cross-border transmission, November 1999.

[8] R. Baldick, The generalized unit commitment problem, IEEE Trans. Power Syst. 10 (1) (1995) 465-475.

[9] J. Batut, A. Renaud, Daily generation scheduling optimization with transmission constraints: a new class of algorithms, IEEE Trans. Power Syst. 7 (3) (1992) 982-989.

[10] J. Shaw, A direct method for security-constrained unit commitment, IEEE Trans. Power Syst. 10 (3) (1995) 1329-1339.

[11] A. Merlin, P. Sandrin, A new method for unit commitment at electricité de France, IEEE Trans. Power Appar. Syst. PAS-102 (5) (1983) $1218-1225$.

[12] F. Zhuang, F.D. Galiana, Towards a more rigorous and practical unit commitment by Lagrangian relaxation, IEEE Trans. Power Syst. 3 (2) (1988) 763-770.

[13] S. Ruzic, N. Rajakovic, A new approach for solving extended unit commitment problem, IEEE Trans. Power Syst. 6 (1) (1991) $269-277$.

[14] S.J. Wang, S.M. Shahidehpour, D.S. Kirschen, S. Mokhtari, G.I. lrisarri, Short-term generation scheduling with transmission and environmental constraints using an augmented Lagrangian relaxation, IEEE Trans. Power Syst. 10 (3) (1995) 1294-1301.

[15] H. Ma, S.M. Shahidehpour, Unit commitment with transmission security and voltage constraints, IEEE Trans. Power Syst. 14 (2) (1999) 757-764.

[16] N. Alguacil, A.J. Conejo, Multiperiod optimal power flow using Benders decomposition, IEEE Trans. Power Syst. 15 (1) (2000) $196-201$.

[17] C.E. Murillo-Sánchez, R.J. Thomas, Thermal unit commitment with a non-linear AC power network model, in: B.F. Hobbs, M.H. Rothkopf, R.P. O’Neill, H. Chao (Eds.), The Next Generation of Electric Power Unit Commitment Models, Kluver Academic Publishers, Boston, 2001, pp. 75-92.

[18] E. Lobato, L. Rouco, T. Gómez, F.M. Echavarren, M.I. Navarrete, R. Casanova, G. López, A practical approach to solve power system constraints with application to the Spanish electricity market, IEEE Trans. Power Syst. 19 (4) (2004) 2029-2037.

[19] J. Martínez-Crespo, J. Usaola, J.L. Fernández, Security-constrained optimal generation scheduling in large-scale power systems, IEEE Trans. Power Syst. 21 (1) (2006) 321-332.

[20] IEEE Reliability Test System, IEEE Trans. Power Appar. Syst. PAS-98 (6) (1979) 2047-2054.

[21] IEEE 118-bus System, available: http://www.edu.es.

[22] A. Brooke, D. Kendrick, A. Meeraus, R. Raman, Release 2. 50 GAMS A User's Guide, GAMS Development Corporation, Washington, DC, 1998.

[23] GAMS/CONOPT, Bagsvaerd, ARKI Consulting and Development A/S, Denmark, 2001.

[24] GAMS/CPLEX 7.0 User's Notes, GAMS Development Corporation, Washington, DC, 2001.

[25] J.M. Arroyo, A.J. Conejo, Optimal response of a thermal unit to an electricity spot market, IEEE Trans. Power Syst. 15 (3) (2000) $1098-1104$.

[26] O. Alsac, B. Stott, Optimal power flow with steady-state security, in: IEEE PES Summer Meeting \& EHV/UHV Conference, Vancouver, May, 1973, pp. $745-751$.

Jorge Martínez-Crespo received his B.S. degree in power engineering from E.T.S. de Ingenieros Industriales de Madrid in 1995 and his Ph.D. degree in electrical engineering from Universidad Carlos III de Madrid in 2004. In 1998, he joined the Department of Electrical Engineering in the Universidad Carlos III de Madrid. His research interests include power system operation, planning and optimization.

Julio Usaola received his B.S. degree and his Ph.D. degree in electrical engineering from E.T.S. de Ingenieros Industriales de Madrid in 1986 and 1990 , respectively. In 1988, he joined the Department of Electrical Engineering in E.T.S. de Ingenieros Industriales de Madrid where he remained until 1994. He is presently an associated professor in the Department of Electrical Engineering in the Universidad Carlos III de Madrid. His research interests are centered on grid integration of wind energy systems and electricity markets.

J.L. Fernández received his B.S. degree and his Ph.D. degree in electrical engineering from E.T.S. de Ingenieros Industriales de Madrid in 1981 and 1987 , respectively. In 1982, he joined the Department of Electrical Engineering in E.T.S. de Ingenieros Industriales de Madrid. He joined Red Eléctrica de España S.A. (REE) in 1989, working at the Network Study Department in the fields of reactive and voltage control, stability and being technical responsible of several Spanish and European funded R\&D projects. He is now at the Regulation Department of REE. 